\title{
Investigation of butterfly check valve with optimum closing performance
}

\author{
WANG XUEFANG, TANG RONGMING, YAO ZHAOHUI \\ YE DINGHUI, YE HONGKAI \\ Dept. of Engineering Mechanics, Tsinghua University, \\ Beijing 100084, China
}

\begin{abstract}
This paper introduces the investigation of butterfly check valve with optimum closing performance, which was developed by Shanghai Petrochemical Complex and Tsinghua University for some feed water systems of power plant and Chemical plant. The valve is consisted of valve body, eccentric shaft disk with $D_{N} 1200$ diameter, governor balance weight hammer and swing damping oil cylinder. All parameters of it have been obtained from optimum $\tau \sim$ t curve for preventing the destruction of systems from waterhammer. The software of optimum calculation has been complied for solving and treating many complex equations and boundary conditions. The paper includes how to determine water impulsive force and momentum on the disk when power fails and pump stops. It is very important and difficult. The experiments of valve turning without the water and valve on industrial systems have been completed and coincide very well with calculation.
\end{abstract}

\section{KEYWORDS}

Butterfly valve, Waterhammer, Optimum performance.

\section{NOMENCLATURE}

$\mathrm{A}=$ area of pipe

$A_{d}=$ area of opening of a valve

$\mathrm{B}=$ pipeline constant $\mathrm{B}=\mathrm{a} / \mathrm{gA}$

$C_{D}=$ orifice discharge coefficient

$\mathrm{d}=$ disk diameter

$\mathrm{D}=$ pipe diameter

e $\quad$ eccentricity of disk

$$
\begin{aligned}
& \mathrm{f} \quad=\text { Darcy-Weisbach friction factor } \\
& F_{F}=\text { friction force on disk } \\
& \mathrm{g}=\text { grivatational acceleration } \\
& G_{g}=\text { weight of balance weight } \\
& G_{v}=\text { weight of valve } \\
& \mathrm{H}=\text { instantaneous piezometric head } \\
& H_{R}=\text { rated pressure head of turbomachine } \\
& \mathrm{I}=\text { polar moment of inertia of rotating } \\
& \quad \text { parts } \\
& L_{V}=\text { arm of force } \mathrm{G}_{\mathrm{v}}
\end{aligned}
$$


$\mathrm{m}=$ mass

$M_{F}=$ friction moment on disk

$N_{R}=$ rated speed of turbomachine

$P_{s 1}, P_{s 2}=$ pressure head

$Q_{R}=$ rated discharge of turbomachine

$\mathbf{R}=$ resistance coefficient of pipe

$\mathrm{t}=$ time

$T_{R}=$ rated torque of turbomachine

$\mathrm{V}=$ instantaneous velocity

$W R^{2} / g=$ polar moment of inertia of rotating parts

$\tau=$ dimenssionless number describing opening of valve

$\delta \quad=$ closing angle of valve disk

$\alpha \quad=$ pipe slope

\section{INTRODUCTION}

Check valve is a one of the important equipments of industrial pipeline systems. It is located on the output of pump. It can prevent reverse flow and protect pump against destruction when pump stops. General check valves are of big resistance, short service life and without closing governor, so they often cause waterhammer and destruction. For many years, we have been investigating valves which can prevent system damage from waterhammer. After swing check valve with property of preventing waterhammer being investigated, recently years the butterfly check valve with $D_{N} 1200$ has been developed for feed water of power plant coolant systems and chemical plant. The investigation of it is introdued in this paper.

The valve consists of valve body, streamlined disk with eccentric shaft, governor balance weight hammer and swing damping oil cylinder. The disk is designed thiner and stronger for smaller resistance.

After comparing with the scales of eccentricity $\frac{d}{4}, \frac{d}{5}, \frac{d}{6}$. by experiment, for getting smaller resistance, $\frac{d}{4}$ eccentricity has been chosen.
All parameters determined according to the optimum $\tau \sim \mathrm{t}$ curve obtained from solving and treating many complex equations and boundary conditions. The paper introduces how to determine water simultaneous impulsive force and moment on the disk when power failure and pump stoping, which is a very importat and very difficult problem.

The software has been complied for calculation. The valve has been manufactured and installed on the systems. It can open rapidly when pump starts and has a less resistance when it operates and realizes two steps adjusted closing speed automatically when pump stops and power fails. The valve can prevent the systems destruction from waterhammer. When valve shuts up its strength and rigidity are safty under the systems pressure. The results of the experiments of both valve on manufacture and valve on industrial pipeline system are coincide very well with the results of calculation.

\section{THE INTRODUCTION OF THE CONSTRUCTURE OF VALVE AND SYSTEMS}

The construction of the valve is shown in Figure 1. (a), (b). The damping oil cylinder with swing cylinder body, is of adjustable damping rod and hole.

The configuration of pipeline systems in which the valve is installed is shown in Figure 2.

\section{ESSENTIAL EQUATIONS AND BOUNDARY CONDITIONS}

\section{Basic Differential Equations for Transient Flow}

\section{Differential Equations:}

$$
\begin{aligned}
& g H_{x}+V V_{t}+V_{t}+\frac{f V|V|}{2 D}=0 \\
& V H_{x}+H_{t}-V \sin \alpha+\frac{a^{2}}{g} V_{x}=0
\end{aligned}
$$




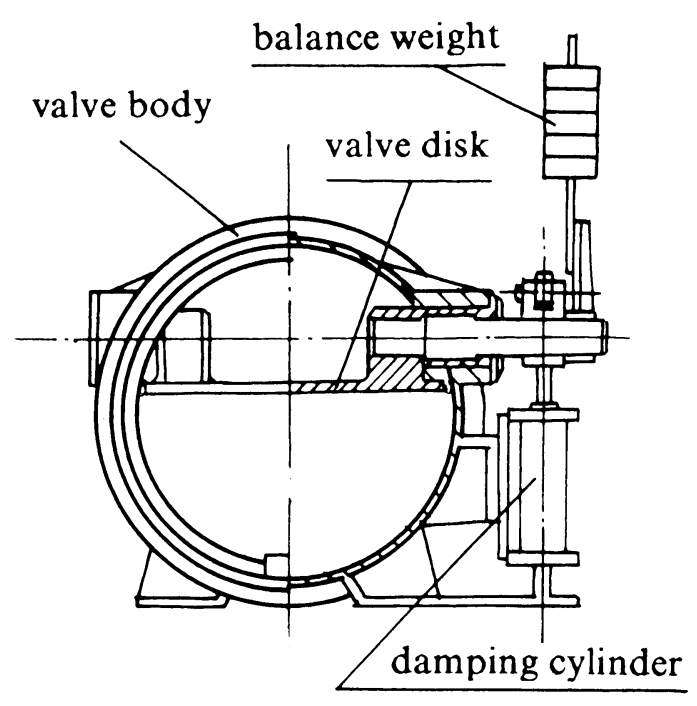

(a) front view

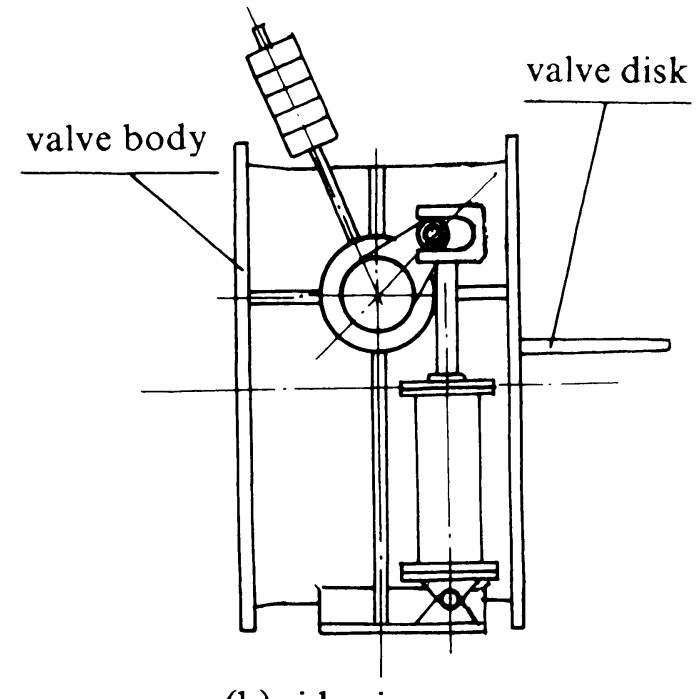

(b) side view

Figure 1. Configuration of the construction of valve. (a) is front view (b) is side view.

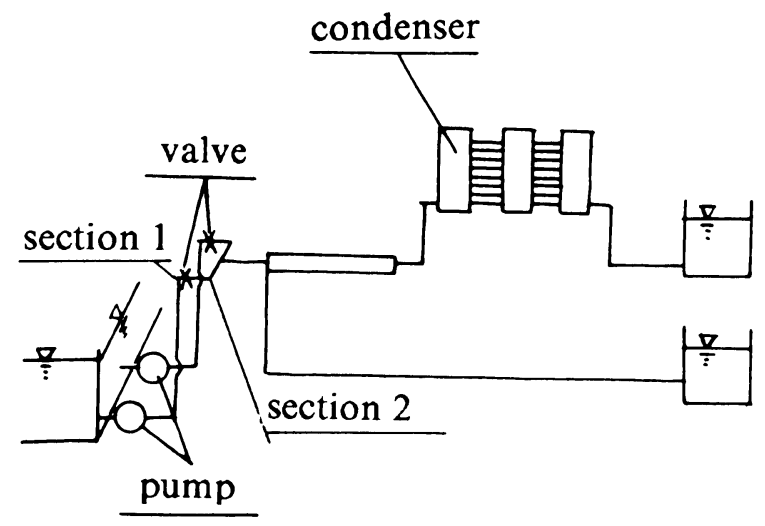

Figure 2. Configuration of pipeline valves installed in pipeline systems
Characteristics Equations:

$$
\begin{aligned}
& C^{+}:\left\{\begin{array}{l}
\frac{g}{a} \frac{d H}{d t}+\frac{d V}{d t}+\frac{f V|V|}{2 D}=0 \\
\frac{d x}{d t}=+a
\end{array}\right. \\
& C^{-}:\left\{\begin{array}{l}
-\frac{g}{a} \frac{d H}{d t}+\frac{d V}{d t}+\frac{f V|V|}{2 D}=0 \\
\frac{d x}{d t}=-a
\end{array}\right.
\end{aligned}
$$

Finite-Difference Equations:

$$
\begin{aligned}
& C^{+}: H_{P i}=C_{P}-B Q_{P i} \\
& C^{-}: H_{P i}=C_{M}-B Q_{P i}
\end{aligned}
$$

in which:

$$
\begin{aligned}
& C_{P}=H_{i-1}+B Q_{i-1}-R Q_{i-1}\left|Q_{i-1}\right| \\
& C_{M}=H_{i+1}-B Q_{i+1}+R Q_{i+1}\left|Q_{i+1}\right|
\end{aligned}
$$

\section{Equation for Pumps in Parallel.}

The Complete Characteristics of the Pump:

$$
\left\{\begin{array}{l}
W H(x)=h /\left(\alpha^{2}+v^{2}\right) \\
W B(x)=\beta /\left(\alpha^{2}+v^{2}\right) \\
x=\pi+\tan ^{-1}\left(\frac{v}{\alpha}\right)
\end{array}\right.
$$

in which $\alpha=\frac{N}{N_{R}}, v=\frac{Q}{Q_{R}}, \beta=\frac{T}{T_{R}}$,

$$
h=\frac{H}{H_{R}} \text {. }
$$

$\mathrm{WH}(\mathrm{x})$ and $\mathrm{WB}(\mathrm{x})$ are the complete characteristics. Pumps are of the same WH(x) and $\mathrm{WB}(\mathrm{x})$ when they are with the same specific speed $n_{S},\left(n_{S}=3.65 N_{R} \sqrt{Q}_{R} / H_{R}^{3 / 4}\right)$. 
Equations for Pumps in Parallel: Pumps in parallel is shown in figure 3.

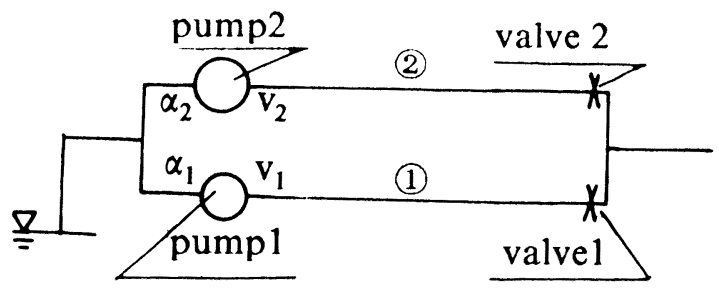

Figure 3. pumps in parallel

For line (1):

$$
\begin{aligned}
F_{11} & =H P M+B S Q v+H_{R_{1}}\left(\alpha_{1}^{2}\right. \\
& \left.+v_{1}^{2}\right) W H\left(x_{1}\right)-\frac{\triangle H_{1} v_{1}\left|v_{1}\right|}{\tau_{1}^{2}}=0 \\
F_{12} & =\left(\alpha_{1}^{2}+v_{1}^{2}\right) W B\left(x_{1}\right)+\beta_{01}-c_{311}\left(\alpha_{01}\right. \\
& \left.-\alpha_{1}\right)=0
\end{aligned}
$$

for line (2):

$$
\begin{aligned}
F_{21} & =H P M+B S Q \cdot v+H_{R_{2}}\left(\alpha_{2}^{2}\right. \\
& \left.+v_{2}^{2}\right) W H\left(x_{2}\right)-\frac{\triangle H_{2} v_{2}\left|v_{2}\right|}{\tau_{2}^{2}}=0 \\
F_{22} & =\left(\alpha_{2}^{2}+v_{2}^{2}\right) W B\left(x_{2}\right)+\beta_{02}-c_{312}\left(\alpha_{02}\right. \\
& \left.-\alpha_{2}\right)=0
\end{aligned}
$$

in which: $v=v_{1}+v_{2}, H P M=C_{P}-C_{M}$, $B S Q=B Q_{R}$

$$
c_{31}=\frac{W R^{2}}{g} \frac{N_{R}}{T_{R}} \frac{\pi}{15 \triangle t}, B=\frac{a}{g A},
$$

a: pressure wave speed

\section{Equations for Valve Moving}

The valve could open, move or close according to $\tau \sim \mathrm{t}$ curve by the action of momentums on disk shaft, such as water impulsive momentum $\mathbf{M}_{\mathrm{H}}$, weight hammer gravity momentum $\mathbf{M}_{\mathrm{G}}$, disk gravity momentum $\mathbf{M}_{v}$, frictional momentum $\mathrm{M}_{\mathrm{F}}$ and piston-rot resistance mo- mentum $\mathbf{M}_{\mathrm{T}}$. Forces acting on the valve are shown in Figure 4.

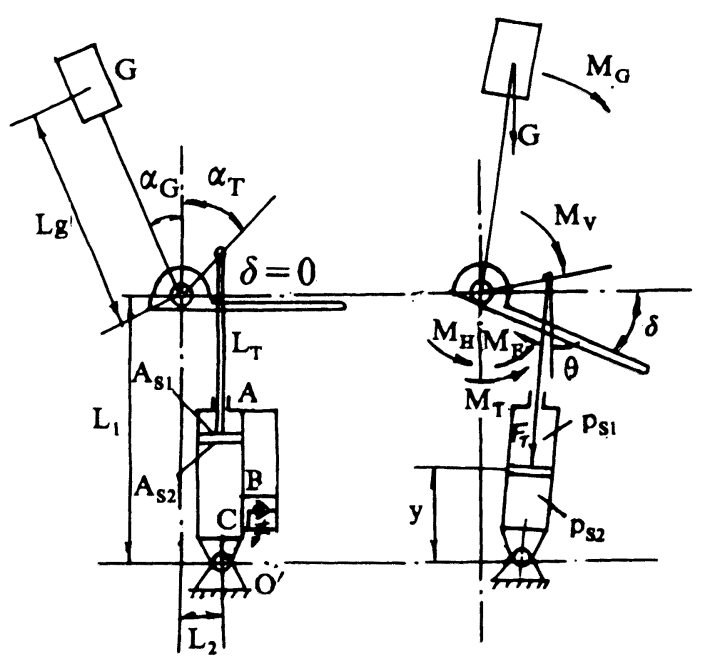

Figure 4. Forces acting on the valve

Continuity Equation of Damping Oil Cylinder:

$$
A_{s 1} \frac{d y}{d t}=C_{d} A_{d}\left[\frac{2}{\rho}\left(p_{s 2}-p_{s 1}\right)\right]^{\frac{1}{2}}
$$

Equation of Piston Motion

$$
\begin{aligned}
m \frac{d^{2} y}{d t^{2}} & =A_{S 2} p_{S 2}-A_{S 1} p_{S 1}+F_{F}-F_{T} \\
& -m g \cos \theta
\end{aligned}
$$

\section{Equation of Disk Rotation}

$$
I \frac{d^{2} \delta}{d t^{2}}=M_{V}+M_{G}+M_{H}-M_{F}-M_{T}
$$

in which:

$$
\begin{aligned}
& M_{V}=L_{V} \cdot G_{V} \cdot \cos \delta \\
& M_{G}=L_{g} \cdot G_{g} \cdot \sin \left(\delta+\alpha_{G}\right) \\
& M_{T}=L_{T} \cdot F_{T} \cdot \sin \left(\delta+\alpha_{T}-\theta\right)
\end{aligned}
$$




$$
\begin{aligned}
F_{H}= & {\left[1.12|(\delta-0.175) / 0.689|^{2.1}\right.} \\
& +0.54] \cdot \rho V^{2} D^{2} \\
M_{H} & =\left[-0.004|(\delta-1.047) / 0.523|^{2.942}\right. \\
& +0.024] \rho V^{2} D^{3},
\end{aligned}
$$

in which:

$\mathbf{M}_{\mathrm{H}}$ gotten from the experiments for symmetry butterfly valve.

For butterfly valve with eccentricy shaft disk, $\mathbf{M}_{\mathrm{H}}$ is as follows:

advance flow: $M_{H}=(e-L) \cdot F_{H}$

reverse flow: $M_{H}=(e+L) \cdot F_{H}$

in which: $L$ is a acting point of $F_{H}$, $\mathrm{e}$ is eccentricity.

\section{Replanishing Equations}

Sixteen equations of condenser and two equations of branch pipes are included.

\section{Boundary Conditions}

Up Stream: sea input pool level

Down Stream: (1) the level of power plant output pool. (2) the level of chemical plant output pool.

\section{AIM OF OPTIMUM $\tau \sim$ t CURVE}

The aim of optimum $\tau \sim \mathrm{t}$ curve is as follows:

The valve could open rapidly when pump starts and is of a smaller resistance when it operates. It has a optimum $\tau \sim \mathrm{t}$ closing curve with smallest waterhammer pressure. The softwares of optimum $\tau \sim \mathrm{t}$ calculation complied for solving the equations and treating the boundary conditions with FORTRAN LANGUAGE. The "Predetermine-Adjust" method has been used to solve equations for obtaining $\delta \sim \mathrm{t}$. The $\tau \sim \mathrm{t}$ curves are obtained from $\delta \sim \mathrm{t}$ curve, $\xi \sim \tau$ relation and $\xi \sim \delta$, Re curve which is gotten from experiments. One $\tau \sim \mathrm{t}$ curve is obtained from one group parameters of valve. According to this $\tau \sim t$, groups of $\mathrm{H}-\mathrm{t}$ at every calculation point of pipes could be got. Comparing many $\tau \sim \mathrm{t}$ and $\mathrm{H} \sim \mathrm{t}$ curves, choosing the Hmin to search the $\tau \sim \mathrm{t}$. That is a optimum $\tau \sim \mathrm{t}$ curve, then all parameters of the valve can be determined.

\section{COMPARE WITH EXPERIMENT}

1. The $\tau \sim t$ curves of the experiments without the water at manufactory coincide with calculations very well

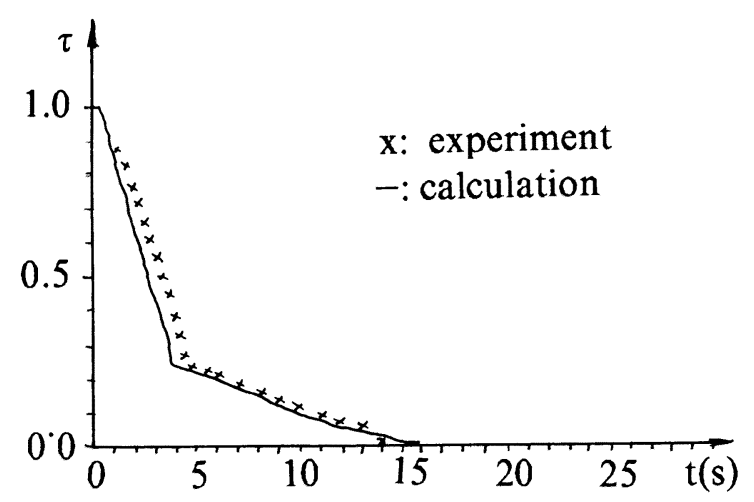

Figure 5. $\tau \sim \mathrm{t}$ curve

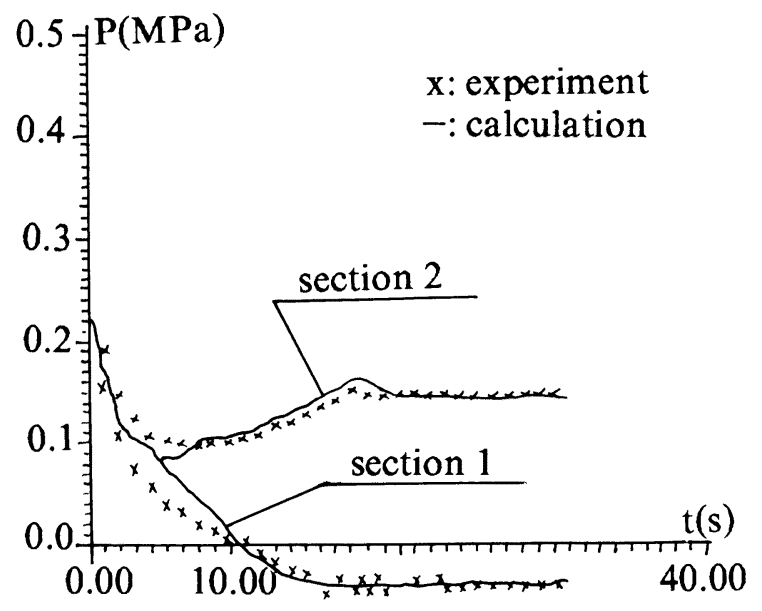

Figure 6. $p \sim t$ curves 
2. The $\tau \sim t$ curves of experiments on industrial pipeline systems with pumps coincide with calculations very well showning in Figure 5.

The $p \sim t$ curves of section 1 and section 2 are shown in Figure 6.

CONCLUSION
1. The investigation and the manufacture of the butterfly check valve are very successful.

2. The method of the optimum $\tau \sim t$ curve is of general significance.

3. The determination of $F_{H}$ and $M_{H}$ is very important and successful.

\section{REFERENCE}

1. E.B.Wylie and V.L.Streeter Fluid Transient, McGraw-Hill, New York, 1983

2. X.F.Wang, H.K.Ye, R.M.Tang A new Type of Check Valve to Guard Againt Waterhammer, first JHPS. 1989, Tokyo, preprints pp401-405. 